\title{
A Hybrid Finite Difference Method for Pricing Two-Asset Double Barrier Options
}

\author{
Y. L. Hsiao, ${ }^{1}$ S. Y. Shen, ${ }^{2}$ and Andrew M. L. Wang ${ }^{3}$ \\ ${ }^{1}$ Department of Finance, National Dong Hwa University, Hualien 97401, Taiwan \\ ${ }^{2}$ Department of Mathematics, National Cheng Kung University, Tainan 70101, Taiwan \\ ${ }^{3}$ Graduate Institute of Finance and Banking, National Cheng Kung University, Tainan 70101, Taiwan
}

Correspondence should be addressed to Y. L. Hsiao; hsiao@mail.ndhu.edu.tw

Received 7 October 2014; Accepted 16 January 2015

Academic Editor: Yan-Wu Wang

Copyright (C) 2015 Y. L. Hsiao et al. This is an open access article distributed under the Creative Commons Attribution License, which permits unrestricted use, distribution, and reproduction in any medium, provided the original work is properly cited.

\begin{abstract}
The pricing of the two-asset double barrier option is modeled as an initial-boundary value problem of the two-dimensional BlackScholes partial differential equation. We use the hybrid finite different method to solve the problem. The hybrid method is a combination of the Laplace transform and a finite difference method. It is more efficient than a traditional finite difference method to obtain a solution without a step-by-step process. The method is implemented on a computer. Two numerical examples are calculated to verify the performance of the hybrid method. In our numerical examples, the convergence rate of the method is approximately two. We conclude that the method is efficient for pricing two-asset barrier options.
\end{abstract}

\section{Introduction}

Pricing financial derivatives is important in financial engineering. Following Black-Scholes arguments [1], pricing a two-asset double barrier option is an initial-boundary value problem of the Black-Scholes partial differential equation (PDE). The Black-Scholes PDE is linear, nonhomogeneous, and parabolic. In this study, we use the hybrid finite difference method to calculate the price of two-asset double barrier option. In order to solve the homogeneous heat equation [24], the method we introduced is first applied in pricing the two-asset double barrier option.

In the literature on this topic, most studies have discussed a one-asset barrier option. However, the probability method is popularly applied in the pricing barrier option; for example, the methods discussed in [5-7]. Some methods have solved the one-dimensional Black-Scholes PDE directly, for example, in [8]. Others have used various simulation methods to calculate the price, for example, in [9]. Pricing a twoasset double barrier option will consume much more computational time as compared to a one-dimensional pricing problem. PDE methods are more efficient than probability methods or simulations. Therefore, PDE methods prevail for two-dimensional problems. There are some publication reports [10-12] that have discussed the two-dimensional problem using PDE methods. Compared to the traditional finite difference method that solves the PDE in a step by step manner, the hybrid finite difference method solved the twodimensional heat equation efficiently [13].

In this study, we introduce the hybrid finite difference method to price the two-asset double barrier option. The outline of this study is arranged as follows. The mathematical problem for two-asset double barrier option is detailed in Section 2. The hybrid method is introduced in Section 3. Section 4 discusses the accuracy of the hybrid method pricing two-asset continuous barrier options. In Section 5, we take a classic numerical example to price the two-asset double barrier option with different immediate rebates. Finally, we draw some conclusions in the last section.

\section{Mathematical Problem for Two-Asset Double Barrier Option}

Two-asset double barrier options are path-dependent derivatives. They are combinations of rainbow options and double 
barrier options. When one of the underlying asset prices touches its upper barrier or lower barrier before expiration, the barrier option will be knock-out. At the same time, the option holder will receive different amounts of immediate rebates. On the other hand, if the underlying asset prices do not touch any barrier, the barrier option will live until maturity and can be executed by their exercise prices.

Under Black-Scholes environments [1], two underlying asset prices follow geometric Brownian motions. The option price can be a function of $x, y$, and time to maturity $\tau$. Let the price be $C(x, y, \tau)$. The function $C$ has to fulfill the BlackScholes equation [14]. The 2D Black-Scholes PDE is

$$
\begin{gathered}
\frac{1}{2} \sigma_{x}^{2} x^{2} C_{x x}+\rho \sigma_{x} \sigma_{y} x y C_{x y}+\frac{1}{2} \sigma_{y}^{2} y^{2} C_{y y} \\
+r x C_{x}+r y C_{y}-r C=C_{\tau}
\end{gathered}
$$

where $x$ and $y$ are two asset prices. $\sigma_{x}$ and $\sigma_{y}$ represent the volatilities of $x$ and $y$, respectively. $r$ is the risk-free rate. $\rho$ is the correlation of two underlying asset prices.

In this study, we set $B_{x}^{u}$ and $B_{x}^{l}$ as the upper and lower barriers with respect to $x$, while $B_{y}^{u}$ and $B_{y}^{l}$ are the upper and lower barriers with respect to $y$. When the underlying asset price $x$ touches the upper barrier $B_{x}^{u}$ or lower barrier $B_{x}^{l}$ at time to maturity $\tau$, the option holder will receive an immediate rebate $f_{x}^{u}(\tau)$ or $f_{x}^{l}(\tau)$, respectively. Similarly, when the underlying asset price $y$ touches the upper barrier $B_{y}^{u}$ or lower barrier $B_{y}^{l}$ at time to maturity $\tau$, the option holder will receive an immediate rebate $f_{y}^{u}(\tau)$ or $f_{y}^{l}(\tau)$, respectively. The functions $f_{x}^{u}(\tau), f_{x}^{l}(\tau), f_{y}^{u}(\tau)$, and $f_{y}^{l}(\tau)$ are continuous or piecewise continuous. If only one of the underlying asset prices touches its barriers at time to maturity $\tau$, the option price will be determined as follows:

$$
\begin{aligned}
& C\left(B_{x}^{u}, y, \tau\right)=f_{x}^{u}(\tau), \quad \text { for } B_{y}^{l}<y<B_{y}^{u}, \\
& C\left(B_{x}^{l}, y, \tau\right)=f_{x}^{l}(\tau), \quad \text { for } B_{y}^{l}<y<B_{y}^{u}, \\
& C\left(x, B_{y}^{u}, \tau\right)=f_{y}^{u}(\tau), \quad \text { for } B_{x}^{l}<x<B_{x}^{u}, \\
& C\left(x, B_{y}^{l}, \tau\right)=f_{y}^{l}(\tau), \quad \text { for } B_{x}^{l}<x<B_{x}^{u},
\end{aligned}
$$

where $f_{x}^{u}(\tau), f_{x}^{l}(\tau), f_{y}^{u}(\tau)$, and $f_{y}^{l}(\tau)$ are the immediate rebate functions with respect to time to maturity $\tau$. Equations (2) are the option's boundary payoffs when the underlying asset prices touch the boundary constraints at time to maturity $\tau$.

On the other hand, if the underlying asset prices $x$ and $y$ never touch their barriers, the option will not be knock-out until maturity and can be executed by their exercise prices. Then, the option payoff at the maturity is the initial condition of the PDE (1),

$$
C(x, y, 0)=\max \left\{x-k_{x}, y-k_{y}, 0\right\},
$$

where $k_{x}$ and $k_{y}$ are the exercise prices with respect to $x$ and $y$, respectively.
The PDE (1), boundary conditions (2) and initial condition (3) compose a well-posed boundary value problem.

\section{The Hybrid Method}

In this section, we use the hybrid method to calculate the solutions of the two-asset double barrier option. The hybrid method is a combination of the Laplace transform and a finite difference method. It is more efficient than a traditional finite difference method to obtain a solution without a step-bystep process since the application of the Laplace transform is used to remove time-dependent terms in the PDE and boundary conditions. We then use the numerical inversion of the Laplace transform to obtain the option price. The Laplace transform is defined as

$$
\widetilde{C}(x, y, p)=\int_{0}^{\infty} e^{-p \tau} C(x, y, \tau) \mathrm{d} \tau .
$$

After using Laplace transform, the time domain will be transformed to the $p$-domain. The derivative of $C$ with respect to $\tau,(\partial / \partial \tau) C$, is transformed to $p \widetilde{C}-C(x, y, 0)$. Therefore, the Black-Scholes PDE (1) becomes

$$
\begin{gathered}
\frac{1}{2} \sigma_{x}^{2} x^{2} \widetilde{C}_{x x}+\rho \sigma_{x} \sigma_{y} x y \widetilde{C}_{x y}+\frac{1}{2} \sigma_{y}^{2} y^{2} \widetilde{C}_{y y}+r x \widetilde{C}_{x} \\
+r y \widetilde{C}_{y}-r \widetilde{C}=p \widetilde{C}-C(x, y, 0),
\end{gathered}
$$

and the boundary conditions (2) are rewritten as follows:

$$
\begin{aligned}
& \widetilde{C}\left(B_{x}^{u}, y, p\right)=\widetilde{f}_{x}^{u}(p), \quad \text { for } B_{y}^{l}<y<B_{y}^{u}, \\
& \widetilde{C}\left(B_{x}^{l}, y, p\right)=\widetilde{f}_{x}^{l}(p), \quad \text { for } B_{y}^{l}<y<B_{y}^{u}, \\
& \widetilde{C}\left(x, B_{y}^{u}, p\right)=\widetilde{f}_{y}^{u}(p), \quad \text { for } B_{x}^{l}<x<B_{x}^{u}, \\
& \widetilde{C}\left(x, B_{y}^{l}, p\right)=\widetilde{f}_{y}^{l}(p), \quad \text { for } B_{x}^{l}<x<B_{x}^{u},
\end{aligned}
$$

where the functions $\tilde{f}_{x}^{u}(p), \tilde{f}_{x}^{l}(p), \tilde{f}_{y}^{u}(p)$, and $\tilde{f}_{y}^{l}(p)$ are the Laplace transform of immediate rebate functions $f_{x}^{u}(\tau)$, $f_{x}^{l}(\tau), f_{y}^{u}(\tau)$, and $f_{y}^{l}(\tau)$, respectively. Equation (5) is an elliptic-type partial differential equation with two variables. It should be noted that the original initial data $C(x, y, 0)$ becomes a part of (5). Equation (5) and boundary conditions (6) compose a well-posed boundary value problem. We employ a finite difference method to solve the boundary value problem.

When we set the increments of $x$ and $y$ as $\Delta x$ and $\Delta y$, respectively, and the numbers for the $x$ and $y$ nodes as $n_{x}$ and $n_{y}$, respectively, then

$$
\Delta x=\frac{B_{x}^{u}-B_{x}^{l}}{n_{x}}, \quad \Delta y=\frac{B_{y}^{u}-B_{y}^{l}}{n_{y}}
$$


and the $x$ and $y$ nodes are presented as

$$
\begin{aligned}
& x_{i}=B_{x}^{l}+i \Delta x, \quad i=0,1,2, \ldots, n_{x}, \\
& y_{j}=B_{y}^{l}+j \Delta y, \quad j=0,1,2, \ldots, n_{y} .
\end{aligned}
$$

Let $\widetilde{C}_{i, j}=\widetilde{C}\left(x_{i}, y_{j}, p\right)$ be the Laplace transform of the barrier option at the point $\left(x_{i}, y_{j}\right)$. Then, the Laplace transform of the boundary conditions (6) at the boundary nodes are

$$
\begin{array}{ll}
\widetilde{C}_{n_{x}, j}=\widetilde{C}\left(B_{x}^{u}, y_{j}, p\right)=\widetilde{f}_{x}^{u}(p), & \text { for } j=1,2, \ldots, n_{y}-1, \\
\widetilde{C}_{0, j}=\widetilde{C}\left(B_{x}^{l}, y_{j}, p\right)=\widetilde{f}_{x}^{l}(p), \quad \text { for } j=1,2, \ldots, n_{y}-1, \\
\widetilde{C}_{i, n_{y}}=\widetilde{C}\left(x_{i}, B_{y}^{u}, p\right)=\widetilde{f}_{y}^{u}(p), \quad \text { for } i=1,2, \ldots, n_{x}-1, \\
\widetilde{C}_{i, 0}=\widetilde{C}\left(x_{i}, B_{y}^{l}, p\right)=\widetilde{f}_{y}^{l}(p), \quad \text { for } i=1,2, \ldots, n_{x}-1 .
\end{array}
$$

Applying the central difference formula provided by [15] to (5), we have the differential equation as follows:

$$
\begin{aligned}
& \frac{1}{2} \sigma_{x}^{2} x_{i}^{2} \frac{\widetilde{C}_{i+1, j}-2 \widetilde{C}_{i, j}+\widetilde{C}_{i-1, j}}{(\Delta x)^{2}} \\
& \quad+\rho \sigma_{x} \sigma_{y} x_{i} y_{j} \\
& \quad \cdot \frac{\widetilde{C}_{i+1, j+1}-\widetilde{C}_{i-1, j+1}-\widetilde{C}_{i+1, j-1}+\widetilde{C}_{i-1, j-1}}{4(\Delta x)(\Delta y)} \\
& +\frac{1}{2} \sigma_{y}^{2} y_{j}^{2} \frac{\widetilde{C}_{i, j+1}-2 \widetilde{C}_{i, j}+\widetilde{C}_{i, j-1}}{(\Delta y)^{2}} \\
& +r x_{i} \frac{\widetilde{C}_{i+1, j}-\widetilde{C}_{i-1, j}}{2(\Delta x)}+r y_{j} \frac{\widetilde{C}_{i, j+1}-\widetilde{C}_{i, j-1}}{2(\Delta y)}-r \widetilde{C}_{i, j} \\
& =p \widetilde{C}_{i, j}-C\left(x_{i}, y_{j}, 0\right), \\
& \quad \text { for } i=1,2, \ldots, n_{x}-1, \quad j=1,2, \ldots, n_{y}-1 .
\end{aligned}
$$

Rearranging the differential equation (10), we obtain

$$
\begin{aligned}
a_{i, j} \widetilde{C}_{i-1, j-1}+b_{i} \widetilde{C}_{i-1, j}+c_{i, j} \widetilde{C}_{i-1, j+1} \\
\quad+d_{j} \widetilde{C}_{i, j-1}+e_{i, j} \widetilde{C}_{i, j}+f_{j} \widetilde{C}_{i, j+1} \\
\quad+c_{i, j} \widetilde{C}_{i+1, j-1}+g_{i} \widetilde{C}_{i+1, j}+a_{i, j} \widetilde{C}_{i+1, j+1} \\
=-C\left(x_{i}, y_{j}, 0\right)
\end{aligned}
$$

where the coefficients of (11) are as follows:

$$
\begin{gathered}
a_{i, j}=\frac{\rho \sigma_{x} \sigma_{y} x_{i} y_{j}}{4 \Delta x \Delta y}, \quad b_{i}=\frac{\sigma_{x}^{2} x_{i}^{2}}{2(\Delta x)^{2}}-\frac{r x_{i}}{2 \Delta x}, \\
c_{i, j}=-\frac{\rho \sigma_{x} \sigma_{y} x_{i} y_{j}}{4 \Delta x \Delta y}=-a_{i, j}, \quad d_{j}=\frac{\sigma_{y}^{2} y_{j}^{2}}{2(\Delta y)^{2}}-\frac{r y_{j}}{2 \Delta y}, \\
e_{i, j}=-\left(\frac{\sigma_{x}^{2} x_{i}^{2}}{(\Delta x)^{2}}+\frac{\sigma_{y}^{2} y_{j}^{2}}{(\Delta y)^{2}}+r+p\right), \\
f_{j}=\frac{\sigma_{y}^{2} y_{j}^{2}}{2(\Delta y)^{2}}+\frac{r y_{j}}{2 \Delta y}, \quad g_{i}=\frac{\sigma_{x}^{2} x_{i}^{2}}{2(\Delta x)^{2}}+\frac{r x_{i}}{2 \Delta x},
\end{gathered}
$$

for $i=1,2, \ldots, n_{x}-1$, and $j=1,2, \ldots, n_{y}-1$.

Combining the differential equation (11) with the Laplace transform of the boundary conditions (9), we have the linear system with a matrix form as follows:

$$
\begin{gathered}
E_{1} \widetilde{\mathbb{C}}_{1}+G_{1} \widetilde{\mathbb{C}}_{2}=\mathbb{F}_{1}, \\
B_{n_{x}-1} \widetilde{\mathbb{C}}_{n_{x}-2}+E_{n_{x}-1} \widetilde{\mathbb{C}}_{n_{x}-1}=\mathbb{F}_{n_{x}-1}, \\
B_{i} \widetilde{\mathbb{C}}_{i-1}+E_{i} \widetilde{\mathbb{C}}_{i}+G_{i} \widetilde{\mathbb{C}}_{i+1}=\mathbb{F}_{i}, \quad \text { for } i=2, \ldots, n_{x}-2,
\end{gathered}
$$

where the square matrices $B_{i}, E_{i}, G_{i}$, and the column matrices $\widetilde{\mathbb{C}}_{i}$ are

$$
B_{i}=\left[\begin{array}{cccccc}
b_{i} & c_{i, 1} & & & & \\
a_{i, 2} & b_{i} & c_{i, 2} & & 0 & \\
& a_{i, 3} & b_{i} & c_{i, 3} & & \\
& & \ddots & \ddots & \ddots & \\
& \mathbf{O} & & a_{i, n_{y}-2} & b_{i} & c_{i, n_{y}-2} \\
& & & & a_{i, n_{y}-1} & b_{i}
\end{array}\right] \text {, }
$$

$$
E_{i}=\left[\begin{array}{cccccc}
e_{i, 1} & f_{1} & & & & \\
d_{2} & e_{i, 2} & f_{2} & & \mathbf{O} & \\
& d_{3} & e_{i, 3} & f_{3} & & \\
& & \ddots & \ddots & \ddots & \\
& \mathbf{O} & & d_{n_{y}-2} & e_{i, n_{y}-2} & f_{n_{y}-2} \\
& & & & d_{n_{y}-1} & e_{i, n_{y}-1}
\end{array}\right] \text {, }
$$$$
G_{i}=\left[\begin{array}{cccccc}
g_{i} & a_{i, 1} & & & & \\
c_{i, 2} & g_{i} & a_{i, 2} & & 0 & \\
& c_{i, 3} & g_{i} & a_{i, 3} & & \\
& & \ddots & \ddots & \ddots & \\
& \mathbf{O} & & c_{i, n_{y}-2} & g_{i} & a_{i, n_{y}-2} \\
& & & & c_{i, n_{y}-1} & g_{i}
\end{array}\right] \text {, }
$$

$$
\begin{aligned}
\widetilde{\mathbb{C}}_{i}= & {\left[\begin{array}{c}
\widetilde{C}_{i, 1} \\
\widetilde{C}_{i, 2} \\
\vdots \\
\widetilde{C}_{i, n_{y}-1}
\end{array}\right], } \\
& \text { for } i=1,2, \ldots, n_{x}-1 .
\end{aligned}
$$

The column matrices $\mathbb{F}_{i}, i=1,2, \ldots, n_{x}-1$ are as follows: 


$$
\begin{gathered}
\mathbb{F}_{1}=\left[\begin{array}{c}
F_{1,1} \\
F_{1,2} \\
\vdots \\
F_{1, n_{y}-2} \\
F_{1, n_{y}-1}
\end{array}\right]=\left[\begin{array}{c}
-C\left(x_{1}, y_{1}, 0\right)-b_{1} \tilde{f}_{x}^{l}(p)-d_{1} \tilde{f}_{y}^{l}(p)+a_{1,1} \min \left\{\tilde{f}_{x}^{l}(p), \tilde{f}_{y}^{l}(p)\right\} \\
-C\left(x_{1}, y_{2}, 0\right)-b_{1} \tilde{f}_{x}^{l}(p) \\
\vdots \\
-C\left(x_{1}, y_{n_{y}-1}, 0\right)-b_{1} \tilde{f}_{x}(p)-f_{n_{y}-1} \tilde{f}_{y}^{u}(p)+c_{1, n_{y}-1} \min \left\{\tilde{f}_{x}^{l}(p), \tilde{f}_{y}^{u}(p)\right\}
\end{array}\right], \\
\mathbb{F}_{n_{x}-1}=\left[\begin{array}{c}
F_{n_{x}-1,1} \\
F_{n_{x}-1,2} \\
\vdots \\
F_{n_{x}-1, n_{y}-2} \\
F_{n_{x}-1, n_{y}-1}
\end{array}\right]=\left[\begin{array}{c}
-C\left(x_{n_{x}-1}, y_{1}, 0\right)-g_{n_{x}-1} \tilde{f}_{x}^{u}(p)-d_{1} \tilde{f}_{y}^{l}(p)+c_{n_{x}-1,1} \min \left\{\tilde{f}_{x}^{u}(p), \tilde{f}_{y}(p)\right\} \\
-C\left(x_{n_{x}-1}, y_{2}, 0\right)-g_{n_{x}-1} \tilde{f}_{x}^{u}(p) \\
\vdots \\
-C\left(x_{n_{x}-1}, y_{n_{y}-1}, 0\right)-g_{n_{x}-1} \tilde{f}_{x}^{u}(p)-f_{n_{y}-1} \tilde{f}_{y}^{u}(p)+a_{n_{x}-1, n_{y}-1} \min \left\{\tilde{f}_{x}^{u}(p), \tilde{f}_{y}^{u}(p)\right\} \\
-C\left(x_{i}, y_{1}, 0\right)-d_{1} \tilde{f}_{y}^{l}(p) \\
-C\left(x_{i}, y_{2}, 0\right) \\
\vdots \\
-C\left(x_{i}, y_{n_{y}-2}, 0\right) \\
F_{i, 1} \\
F_{i, 2} \\
\vdots \\
F_{i, n_{y}-2} \\
F_{i, n_{y}-1}
\end{array}\right]=\left[\begin{array}{c}
\left.x_{n_{x}-1}, y_{n_{y}-2}, 0\right)-\tilde{f}_{x}^{u}(p) \\
-C\left(x_{i}, y_{n_{y}-1}, 0\right)-f_{n_{y}-1} \tilde{f}_{y}^{u}(p)
\end{array}\right], \quad \text { for } i=2, \ldots, n_{x}-2 .
\end{gathered}
$$

Therefore, we can represent the linear system (13) to a simple matrix form as

$$
[\mathbb{K}][\widetilde{\mathbb{C}}]=[\mathbb{F}],
$$

where $[\mathbb{K}]$ is an $\left(n_{x}-1\right)\left(n_{y}-1\right) \times\left(n_{x}-1\right)\left(n_{y}-1\right)$ square matrix with $B_{i}, E_{i}$, and $G_{i}$. [ $\left.\widetilde{\mathbb{C}}\right]$ is an $\left(n_{x}-1\right)\left(n_{y}-1\right) \times 1$ column matrix with $\widetilde{\mathbb{C}}_{i}$, and the matrix $[\widetilde{\mathbb{C}}]$ represents the Laplace transform value of the barrier option with respect to all nodes $\left(x_{i}, y_{j}\right)$ in the transformed domain. $[\mathbb{F}]$ is a column matrix with $\mathbb{F}_{i}$, which is a combination of initial and boundary conditions. The dimension of matrix $[\mathbb{F}]$ is the same as the matrix $[\widetilde{\mathbb{C}}]$. The matrices $[\mathbb{K}],[\widetilde{\mathbb{C}}]$ and $[\mathbb{F}]$ are described as follows:

$$
\begin{gathered}
{[\mathbb{K}]=\left[\begin{array}{cccccc}
E_{1} & G_{1} & & & & \\
B_{2} & E_{2} & G_{2} & & \mathbf{O} & \\
& B_{3} & E_{3} & G_{3} & & \\
& & \ddots & \ddots & \ddots & \\
& \mathbf{O} & & B_{n_{x}-2} & E_{n_{x}-2} & G_{n_{x}-2} \\
& & & B_{n_{x}-1} & E_{n_{x}-1}
\end{array}\right],} \\
{[\widetilde{\mathbb{C}}]=\left[\begin{array}{c}
\widetilde{\mathbb{C}}_{1} \\
\widetilde{\mathbb{C}}_{2} \\
\vdots \\
\widetilde{\mathbb{C}}_{n_{x}-1}
\end{array}\right],}
\end{gathered}
$$

After using the double Gaussian elimination algorithm procedures in the linear system (16), we can obtain the Laplace transform value $\widetilde{C}_{i, j}$ of the two-asset double barrier option with respect to any point $\left(x_{i}, y_{j}\right)$ in the transformed domain. We then use the numerical inversion of the Laplace transform from Honig and Hirdes [16] and Durbin [17] to obtain the option price $C_{i, j}$ in the original domain. In this method, the inversion approximation formula (18) is used:

$$
\begin{aligned}
C\left(x_{i}, y_{j}, \tau\right) & =\frac{e^{v \tau}}{T}\left[-\frac{1}{2} \operatorname{Re}\left\{\widetilde{C}_{i, j}(v)\right\}+\sum_{k=0}^{\infty} \operatorname{Re}\left\{\widetilde{C}_{i, j}\left(v+i \frac{k \pi}{T}\right)\right\}\right. \\
& \cdot \cos \left(\frac{k \pi}{T} \tau\right)-\sum_{k=0}^{\infty} \operatorname{Im}\left\{\widetilde{C}_{i, j}\left(v+i \frac{k \pi}{T}\right)\right\} \\
& \left.\cdot \sin \left(\frac{k \pi}{T} \tau\right)\right],
\end{aligned}
$$

where $v$ is a constant value, $v \in \mathbf{R}$, and $0<\tau<T$. Here, we set the constant number $v=\left(\Delta x / \sigma_{x}\right)\left(\Delta y / \sigma_{y}\right)(1 / T)$.

Since we can only calculate finite terms, we truncate (18) as follows:

$$
\begin{aligned}
C\left(x_{i}, y_{j}, \tau\right) \\
=\frac{e^{v \tau}}{T}\left[-\frac{1}{2} \operatorname{Re}\left\{\widetilde{C}_{i, j}(v)\right\}+\sum_{k=0}^{n} \operatorname{Re}\left\{\widetilde{C}_{i, j}\left(v+i \frac{k \pi}{T}\right)\right\}\right. \\
\cdot \cos \left(\frac{k \pi}{T} \tau\right)-\sum_{k=0}^{n} \operatorname{Im}\left\{\widetilde{C}_{i, j}\left(v+i \frac{k \pi}{T}\right)\right\} \\
\left.\cdot \sin \left(\frac{k \pi}{T} \tau\right)\right],
\end{aligned}
$$


TABLE 1: Accuracy of the valuations of the two-asset double barrier options using the hybrid method.

\begin{tabular}{|c|c|c|c|c|c|}
\hline Current underlying asset prices & $n_{x} \times n_{y}$ & $C_{n}$ & $C_{2 n}-C_{n}$ & $C_{64}-C_{n}$ & $\frac{\left|C_{64}-C_{n}\right|}{C_{64}}$ \\
\hline \multirow{5}{*}{$\begin{array}{l}s_{1}=12 \\
s_{2}=15\end{array}$} & $4 \times 4$ & 0.225100 & 0.087465 & 0.124744 & 0.356570 \\
\hline & $8 \times 8$ & 0.312565 & 0.027761 & 0.037279 & 0.106559 \\
\hline & $16 \times 16$ & 0.340326 & 0.007581 & 0.009518 & 0.027206 \\
\hline & $32 \times 32$ & 0.347907 & 0.001937 & 0.001937 & 0.005537 \\
\hline & $64 \times 64$ & 0.349844 & * & * & $*$ \\
\hline \multirow{5}{*}{$\begin{array}{l}s_{1}=16 \\
s_{2}=20\end{array}$} & $4 \times 4$ & 0.996184 & 0.364442 & 0.468376 & 0.319807 \\
\hline & $8 \times 8$ & 1.360626 & 0.080328 & 0.103934 & 0.070966 \\
\hline & $16 \times 16$ & 1.440954 & 0.018920 & 0.023606 & 0.016118 \\
\hline & $32 \times 32$ & 1.459874 & 0.004686 & 0.004686 & 0.003200 \\
\hline & $64 \times 64$ & 1.464560 & * & * & * \\
\hline \multirow{5}{*}{$\begin{array}{l}s_{1}=20 \\
s_{2}=25\end{array}$} & $4 \times 4$ & 1.141040 & 0.036247 & 0.048109 & 0.040457 \\
\hline & $8 \times 8$ & 1.177287 & 0.008428 & 0.011862 & 0.009975 \\
\hline & $16 \times 16$ & 1.185715 & 0.002704 & 0.003434 & 0.002888 \\
\hline & $32 \times 32$ & 1.188419 & 0.000730 & 0.000730 & 0.000614 \\
\hline & $64 \times 64$ & 1.189149 & * & * & * \\
\hline
\end{tabular}

Option parameters: $\left(B_{x}^{l}, B_{x}^{u}\right)=(8,24),\left(B_{y}^{l}, B_{y}^{u}\right)=(10,30), k_{x}=16, k_{y}=20,\left(R_{x}^{l}, R_{x}^{u}\right)=(0,0),\left(R_{y}^{l}, R_{y}^{u}\right)=(0,0), \sigma_{x}=0.3, \sigma_{y}=0.2, \rho=0.3, r=0.01$, and $T=1$ year.

for $0<\tau<T$. Considering the truncation error in (19),

$$
\text { truncation error }<\frac{e^{v T}}{2 T}\left|\widetilde{C}_{i, j}\left(v+i \frac{n \pi}{T}\right)\right| \text {, }
$$

and $\left|\widetilde{C}_{i, j}(v+i(k \pi / T))\right|$ decreases monotonically to zero [17]; then we have

$$
\left|\widetilde{C}_{i, j}\left(v+i \frac{n \pi}{T}\right)\right| \leq \frac{2 \varepsilon T}{e^{v T}}
$$

for any $\varepsilon>0$. That is, when we take any tolerance value $\varepsilon$, there exists a number $n$ to satisfy (21). The inequality (21) is used to determine $n$. For the inverse Laplace transformation, the discrete Laplace transform points are $p_{k}=v+i(k \pi / T)$, $k=0,1,2, \ldots, n$.

\section{Accuracy for the Hybrid Method}

This section provides two numerical examples to verify the performance of the hybrid finite difference method. To assess the validity of the hybrid method for pricing the two-asset double barrier option, we compare the results under various numbers of nodes with the most accurate case.

For example, we set the pair of lower and upper boundaries for the first asset as $\left(B_{x}^{l}, B_{x}^{u}\right)=(8,24)$ and that for the second asset as $\left(B_{y}^{l}, B_{y}^{u}\right)=(10,30)$. The exercise prices of the first and second assets are $k_{x}=16$ and $k_{y}=20$. The volatilities of the first and second assets' prices are $\sigma_{x}=0.3$ and $\sigma_{y}=$ 0.2 . The correlation between the two assets' prices is 0.3 , while the risk free rate is 0.01 . The time to maturity $T$ of the option contract is one year. Since the construct of the inversion approximation formula for the Laplace transform is $0<\tau<$ $T$, we solve a closed solution before the time to maturity $T$. In our numerical examples, we control the computational error of $10^{-5}$ and solve the solution of time to maturity $T-0.00001$ as our construct value.

Table 1 shows the performance of the hybrid method for pricing the two-asset double barrier option out of the money (the case of $s_{1}=12$ and $s_{2}=15$ ), at the money (the case of $s_{1}=16$ and $s_{2}=20$ ), and in the money (the case of $s_{1}=20$ and $s_{2}=25$ ), respectively. When we compare each pricing result with the most accurate case, it can be seen that the numerical errors and relative errors both have reached at least a significant digit level of 2 in all scenarios with the number of nodes $16 \times 16$. From the fifth column of Table 1 , we can see that the error results become one fourth when the number of nodes doubles at each dimension. The order of the method is about two in our experimental results.

Additionally, in order to test the validity of the hybrid method for pricing the two-asset single barrier option, we extend boundary conditions to set two assets to only have the upper barriers $B_{x}^{u}=16$ and $B_{y}^{u}=20$, while there are no lower barriers imposed in two assets. The exercise prices with respect to the first asset and second asset are $k_{x}=8$ and $k_{y}=10$, while we set the volatilities $\sigma_{x}=0.3$ and $\sigma_{y}=0.2$. The correlation between the two asset prices is 0.3 , and the risk free rate is 0.01 . The time to maturity of the barrier option is one year.

Table 2 shows the performance of the hybrid method for pricing the two-asset up-and-out barrier options out of the money $\left(s_{1}=4, s_{2}=5\right)$, at the money $\left(s_{1}=8, s_{2}=10\right)$, and in the money $\left(s_{1}=12, s_{2}=15\right)$, respectively. In this example, the convergence performance is the best when the barrier option is in the money. It can be seen that the numerical errors for pricing the barrier option have reached at least a significant digit level of 2 in all scenarios, while the number of the nodes is 16 by 16 . 
TABLE 2: Accuracy of the valuations of the two-asset single barrier options using the hybrid method.

\begin{tabular}{|c|c|c|c|c|c|}
\hline Current underlying asset prices & $n_{x} \times n_{y}$ & $C_{n}$ & $C_{2 n}-C_{n}$ & $C_{64}-C_{n}$ & $\frac{\left|C_{64}-C_{n}\right|}{C_{64}}$ \\
\hline \multirow{5}{*}{$\begin{array}{l}s_{1}=4 \\
s_{2}=5\end{array}$} & $4 \times 4$ & 0.019511 & -0.007309 & -0.012745 & 1.883683 \\
\hline & $8 \times 8$ & 0.012202 & -0.003689 & -0.005436 & 0.803429 \\
\hline & $16 \times 16$ & 0.008513 & -0.001371 & -0.001747 & 0.258203 \\
\hline & $32 \times 32$ & 0.007142 & -0.000376 & -0.000376 & 0.055572 \\
\hline & $64 \times 64$ & 0.006766 & * & * & * \\
\hline \multirow{5}{*}{$\begin{array}{l}s_{1}=8 \\
s_{2}=10\end{array}$} & $4 \times 4$ & 0.680657 & 0.468757 & 0.683742 & 0.501131 \\
\hline & $8 \times 8$ & 1.149414 & 0.171159 & 0.214985 & 0.157568 \\
\hline & $16 \times 16$ & 1.320573 & 0.035646 & 0.043826 & 0.032121 \\
\hline & $32 \times 32$ & 1.356219 & 0.008180 & 0.008180 & 0.005995 \\
\hline & $64 \times 64$ & 1.364399 & * & * & * \\
\hline \multirow{5}{*}{$\begin{array}{l}s_{1}=12 \\
s_{2}=15\end{array}$} & $4 \times 4$ & 2.708195 & 0.138637 & 0.147685 & 0.051713 \\
\hline & $8 \times 8$ & 2.846832 & 0.006731 & 0.009048 & 0.003168 \\
\hline & $16 \times 16$ & 2.853563 & 0.001831 & 0.002317 & 0.000811 \\
\hline & $32 \times 32$ & 2.855394 & 0.000486 & 0.000486 & 0.000170 \\
\hline & $64 \times 64$ & 2.855880 & * & * & * \\
\hline
\end{tabular}

Option parameters: $B_{x}^{u}=16, B_{y}^{u}=20, k_{x}=8, k_{y}=10, \sigma_{x}=0.3, \sigma_{y}=0.2, R_{x}^{u}=0, R_{y}^{u}=0, \rho=0.3, r=0.01$, and $T=1$ year.

TABLE 3: The valuations of the two-asset double barrier options with various immediate rebates.

\begin{tabular}{|c|c|c|c|c|c|c|}
\hline \multirow{2}{*}{$R_{y}^{u}$} & \multicolumn{6}{|c|}{$R_{x}^{u}$} \\
\hline & 5 & 10 & 15 & 20 & 25 & 30 \\
\hline 5 & 3.322664 & 4.256101 & 5.189538 & 6.122976 & 7.056413 & 7.989850 \\
\hline 10 & 4.634732 & 5.567645 & 6.501082 & 7.434519 & 8.367956 & 9.301394 \\
\hline 15 & 5.946800 & 6.879713 & 7.812626 & 8.746063 & 9.679500 & 10.612937 \\
\hline 20 & 7.258868 & 8.191781 & 9.124694 & 10.057607 & 10.991044 & 11.924481 \\
\hline 25 & 8.570936 & 9.503849 & 10.436762 & 11.369675 & 12.302587 & 13.236025 \\
\hline 30 & 9.883004 & 10.815917 & 11.748830 & 12.681743 & 13.614655 & 14.547568 \\
\hline
\end{tabular}

Option parameters: $s_{1}=45.6, s_{2}=42.6, k_{x}=45.6, k_{y}=42.6,\left(B_{x}^{l}, B_{x}^{u}\right)=(22.8,68.4),\left(B_{y}^{l}, B_{y}^{u}\right)=(21.3,63.9), R_{x}^{l}=0, R_{y}^{l}=0, \rho=0.3778, r=0.0154$, and $T=1$ year. The number of nodes: $n_{x} \times n_{y}=64 \times 64$.

From Tables 1 and 2, we can see that the convergent order is about two whether the boundary conditions have single barriers or double barriers when we double the number of the nodes.

\section{A Numerical Example in Financial Engineering}

In this section, a numerical example for pricing the twoasset double barrier option is used to demonstrate the effect of immediate rebates while common assumptions set immediate rebates to be zero.

In this case, we set the current underlying asset prices, and the exercise prices with respect to the first and second assets are 45.6 and 42.6 , respectively, so that the case $\left(s_{1}=\right.$ $\left.k_{x}=45.6, s_{2}=k_{y}=42.6\right)$ is called at-the-money. The upper and lower barriers with respect to the first and second assets are $\left(B_{x}^{l}, B_{x}^{u}\right)=(22.8,68.4)$ and $\left(B_{y}^{l}, B_{y}^{u}\right)=(21.3,63.9)$. The correlation between the two asset prices is 0.3778 , and the risk free rate is 0.0154 . The time to maturity of the barrier option is one year.
The amounts of immediate rebates are important factors with regard to the barrier option price. We compute the pricing of the two-asset double barrier option with various upper immediate rebates, $R_{x}^{u}$ and $R_{y}^{u}$. The option holder will receive the upper immediate rebates when the asset prices touch their upper barriers. On the other hand, the lower immediate rebates are set to be zero in this case. The results of pricing the two-asset double barrier option with various upper immediate rebates are shown in Table 3 via the hybrid finite difference method with nodes $64 \times 64$. We can see that the barrier option price increases significantly with the amount of immediate rebates.

\section{Conclusions}

Pricing financial derivatives is a mathematical problem in financial engineering. Through the hybrid finite difference method, which is a combination of the Laplace transform and a finite difference method, we solve the two-dimensional Black-Scholes partial deferential equation to price the twoasset double barrier option. The method is first applied in 
pricing the two-asset double barrier option although the hybrid finite difference method is applied to solve the heat conduct problem for a while. Then, we take a classic example of a two-asset double barrier option with immediate rebates in our study, and we find that the price of the two-asset double barrier option increases with the immediate rebates. The method is second order. Therefore, we can conclude that the method is efficient for pricing two-asset barrier options.

\section{Conflict of Interests}

The authors declare that there is no conflict of interests regarding the publication of this paper.

\section{References}

[1] F. Black and M. Scholes, "The pricing of options and corporate liabilities," Journal of Political Economy, vol. 81, no. 3, pp. 637654, 1973.

[2] H. T. Chen, T. M. Chen, and C. K. Chen, "Hybrid Laplace transform/finite element method for one-dimensional transient heat conduction problems," Computer Methods in Applied Mechanics and Engineering, vol. 63, no. 1, pp. 83-95, 1987.

[3] H. T. Chen and C. K. Chen, "Hybrid Laplace transform/finite difference method for transient heat conduction problems," International Journal for Numerical Methods in Engineering, vol. 26, no. 6, pp. 1433-1447, 1988.

[4] H. T. Chen and C. K. Chen, "Hybrid laplace transform/finite difference method for two-dimensional transient heat conduction," Journal of Thermophysics and Heat Transfer, vol. 2, no. 1, pp. 31-36, 1998.

[5] N. Kunitomo and M. Ikeda, "Pricing options with curved boundaries," Mathematical Finance, vol. 2, no. 4, pp. 275-298, 1992.

[6] H. Geman and M. Yor, "Pricing and hedging double-barrier options: a probabilistic approach," Mathematical Finance, vol. 6, no. 4, pp. 365-378, 1996.

[7] A. Pelsser, "Pricing double barrier options using Laplace transforms," Finance and Stochastics, vol. 4, no. 1, pp. 95-104, 2000.

[8] A. M. L. Wang, Y. H. Liu, and Y. L. Hsiao, "Barrier option pricing: a hybrid method approach," Quantitative Finance, vol. 9, no. 3, pp. 341-352, 2009.

[9] S. M. Ross and S. Ghamami, "Efficient Monte Carlo barrier option pricing when the underlying security price follows a jump-diffusion process," The Journal of Derivatives, vol. 17, no. 3, pp. 45-52, 2010.

[10] T. H. F. Cheuk and T. C. F. Vorst, "Complex barrier options," The Journal of Derivatives, vol. 4, no. 1, pp. 8-22, 1996.

[11] P. Ritchken, "On pricing barrier options," The Journal of Derivatives, vol. 3, no. 2, pp. 19-28, 1995.

[12] A. M. L. Wang and Y. L. Hsiao, "A PDE approach to value the discrete two-asset barrier option," Journal of Financial Studies, vol. 14, no. 3, pp. 1-33, 2006.

[13] W. Chong, R. Tramontini, and L. P. Specht, "Application of the Laplace transform and its numerical inversion to temperature profile of a two-layer pavement under site conditions," Numerical Heat Transfer Part A: Applications, vol. 55, no. 11, pp. 10041018, 2009.

[14] J. C. Hull, Options, Futures, and Other Derivatives, Prentice Hall, 5th edition, 2002.
[15] D. J. Duffy, Finite Difference Methods in Financial Engineering, John Wiley \& Sons, Chichester, UK, 2006.

[16] G. Honig and U. Hirdes, "A method for the numerical inversion of Laplace transforms," Journal of Computational and Applied Mathematics, vol. 10, no. 1, pp. 113-132, 1984.

[17] F. Durbin, "Numerical inversion of Laplace transforms: an efficient improvement to Dubner and Abate's method," The Computer Journal, vol. 17, no. 4, pp. 371-376, 1974. 


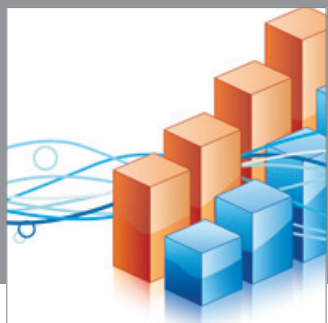

Advances in

Operations Research

mansans

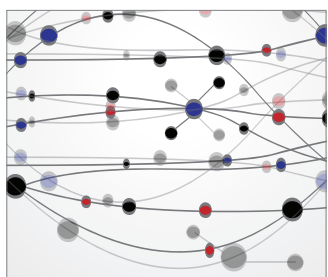

The Scientific World Journal
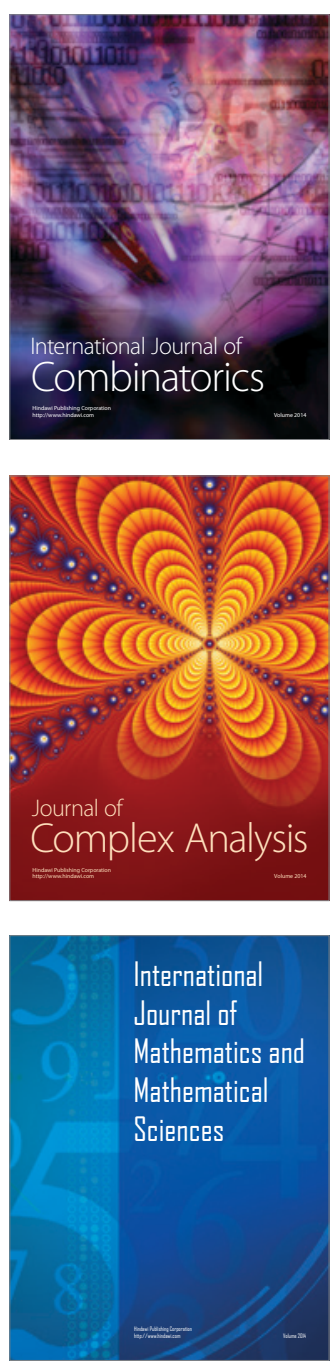
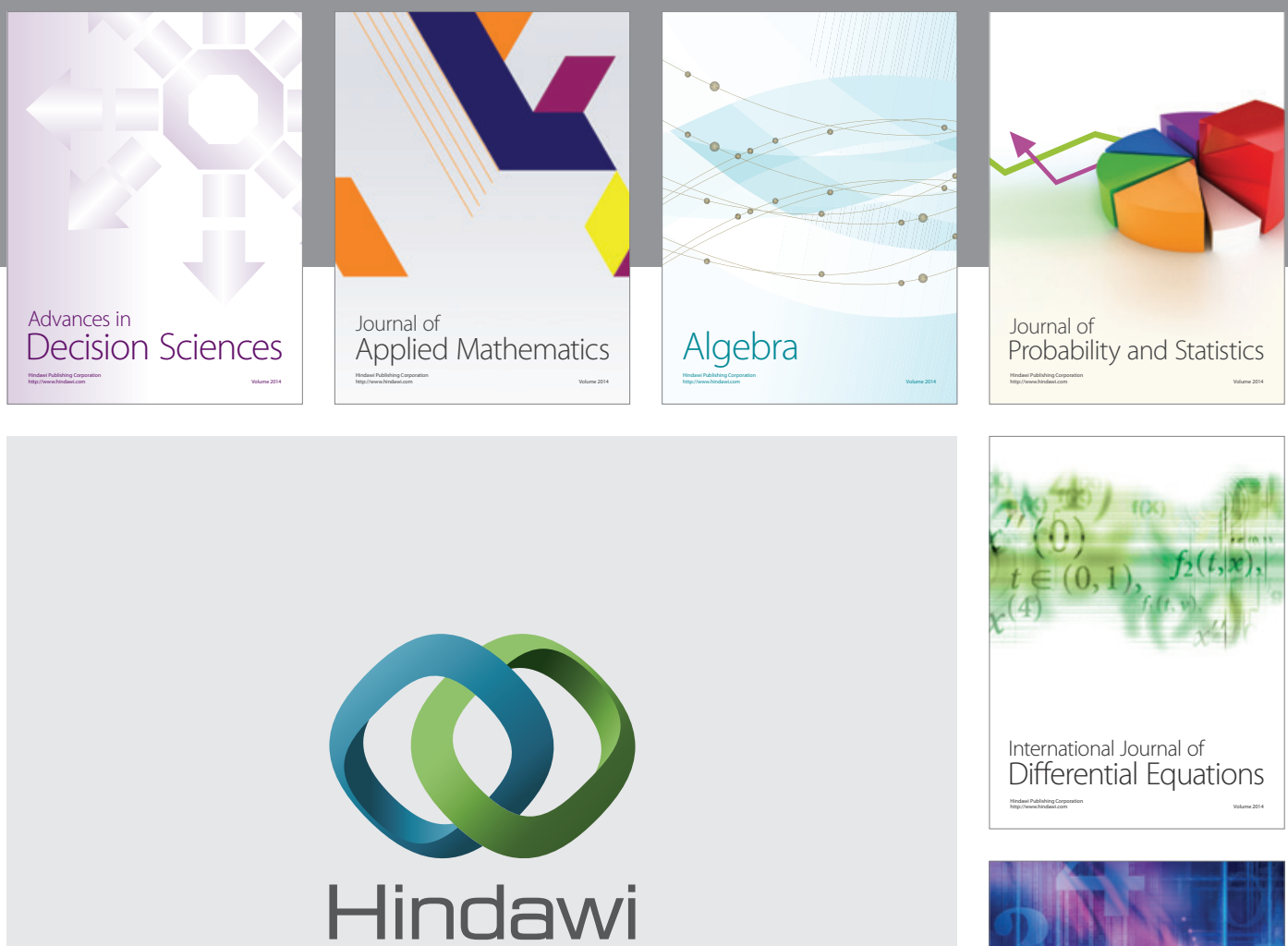

Submit your manuscripts at http://www.hindawi.com
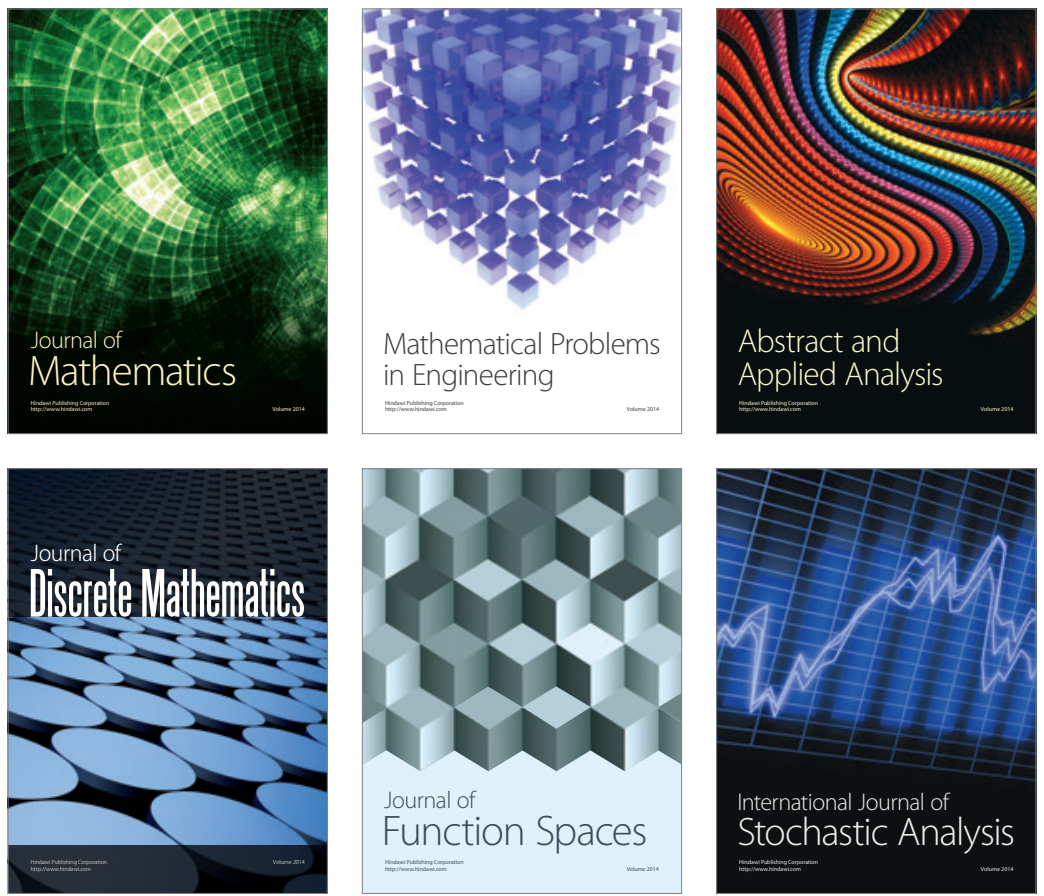

Journal of

Function Spaces

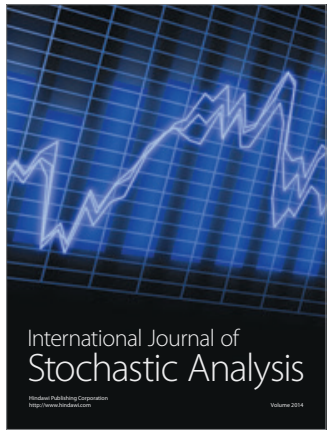

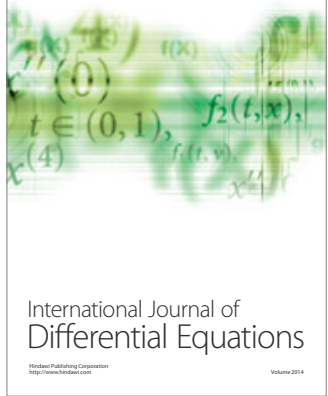
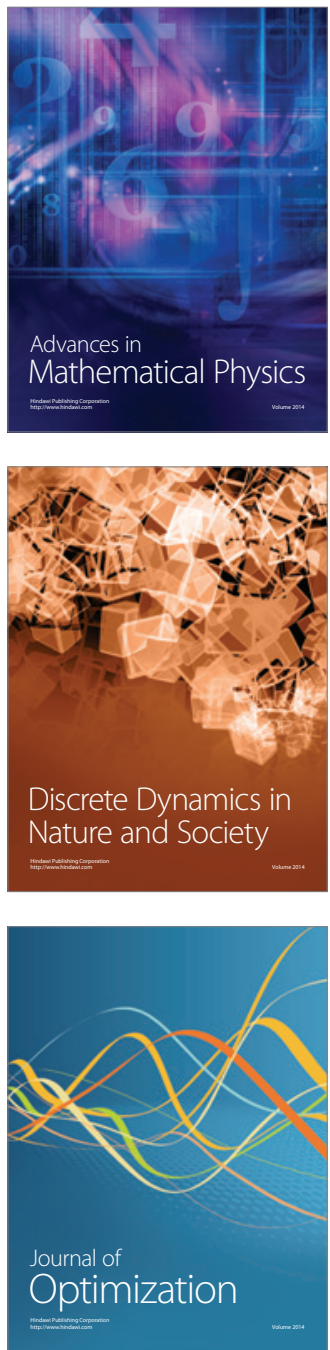